Décadrages Décadrages

cinéma, à travers champs Cinéma, à travers champs

8-9 | 2006

Le monde de Star Wars

\title{
Entre opéra wagnérien et culture de masse : l'univers musical de Star Wars
}

Laurent Guido

\section{(2) OpenEdition}

1 Journals

Édition électronique

URL : https://journals.openedition.org/decadrages/280

DOI : $10.4000 /$ decadrages. 280

ISSN : 2297-5977

\section{Éditeur}

Association Décadrages

\section{Édition imprimée}

Date de publication : 10 octobre 2006

Pagination : $52-75$

ISBN : 978-2-9700582-4-3

ISSN : 2235-7823

\section{Référence électronique}

Laurent Guido, "Entre opéra wagnérien et culture de masse : l'univers musical de Star Wars », Décadrages [En ligne], 8-9 | 2006, mis en ligne le 04 février 2014, consulté le 04 avril 2022. URL : http:// journals.openedition.org/decadrages/280 ; DOI : https://doi.org/10.4000/decadrages.280 


\section{Entre opéra wagnérien et culture de masse :}

\section{l'univers musical de Star Wars}

\section{par Laurent Guido}

1 Les quatre opéras ont été composés et terminés entre 1848 et 1874.

2 Voir notamment David Schroeder, Cinema's Illusions, Opera's Allure. The Operatic Impulse in Film, Continuum, New York/Londres, pp. 215219; la page de Kristian Eversen, "The Star Wars Series and Wagner's Ring Structural, Thematic and Musical Connections", www.trell. org/wagner/starwars.html, ainsi que Vienna Online, Volume 2, Issue 1a, July/August 1999 (www.geocities.com/viennaonline/feature/ starwars.html).
La référence à l'opéra fait partie des lieux communs véhiculés dans les discours promotionnels et critiques autour de Star Wars en raison de l'alliance spectaculaire et imposante qui s'y déploie entre récit épique, machinerie et sonorités orchestrales. Cette perception d'un caractère opératique dans les six films produits par George Lucas entre 1977 et 2005 s'ancre le plus souvent dans une comparaison avec l'œuvre de Richard Wagner, en particulier sa tétralogie L'Anneau du Nibelung. Un siècle après la première présentation de ce cycle d'opéras dans sa chronologie diégétique (en 1876, moment où se produit la synthèse d'une vingtaine d'années de travail créatif $\mathbf{1}$ ), l'industrie hollywoodienne aurait ainsi engendré une œuvre analogue à celle du compositeur de Bayreuth. Il est certes possible d'effectuer ce rapprochement d'une manière littérale en signalant les multiples ressemblances entre les sagas scénique et cinématographique, aussi bien sur le plan des structures dramatiques qu'au niveau des univers représentés ou encore de différents thèmes musicaux ${ }^{2}$. Mais il me paraît plus intéressant d'envisager cette problématique au-delà des citations et des échos directs, plus ou moins avérés, en considérant avant tout la postérité de certains principes généraux de l'esthétique wagnérienne, tels que la constitution d'un vaste univers à partir de l'adaptation/reformulation de bases mythologiques, ou encore le recours à la technique du leitmotiv pour assurer musicalement la compréhension la plus immédiate possible des tensions dramatiques et des conflits philosophiques fondamentaux du récit.

\section{De Wagner à Hollywood: le recours au leitmotiv}

Ancrées essentiellement dans l'idiome post-romantique de Mahler, Bruckner ou Richard Strauss tout en s'inspirant quelquefois de morceaux de Tchaïkovski, Holst ou Stravinsky, les partitions signées par John Williams (fig. 1) pour les six films de Star Wars font un usage intensif du leitmotiv. Systématisé par Wagner dans ses opéras, ce procédé 
a marqué depuis longtemps les pratiques de la musique de cinéma. Dès les années 1910, la présence d'une musique orchestrale soigneusement adaptée au film, par exemple pour des ouvres monumentales comme Cabiria (Giovanni Pastrone, 1914) ou Naissance d'une nation (The Birth of a Nation, D. W. Griffith, 1915), a obéi à deux logiques complémentaires: d'une part la volonté de dégager une puissance destinée à renforcer la participation émotionnelle des spectateurs, sur le modèle explicite de l'opéra wagnérien; d'autre part, le souci de renforcer la standardisation vers le long métrage de fiction, basé sur la continuité spatio-temporelle et l'absorption diégétique ${ }^{3}$. A l'instar des pratiques de montage fondées sur les raccords, la musique a été mise au service des impératifs du récit et de la psychologie des personnages, notamment via le recours à la pratique homogénéisante du leitmotiv. Cette association de motifs mélodiques à des protagonistes, à des valeurs ou à des sentiments révèle combien la musique clarifie les grands axes narratifs et émotionnels du drame visuel, engageant potentiellement de multiples effets de reprises et de variations.

Après l'arrivée du parlant, cette conception de la musique de film s'est généralisée à Hollywood. En témoignent les partitions de compositeurs directement issus de la culture postromantique européenne, comme Max Steiner, Erich Wolfgang Korngold ou Franz Waxman. Sous l'impulsion de producteurs soucieux de légitimation artistique (sur le mode de la Warner au milieu des thirties, multipliant les adaptations de romans historiques à succès pour égaler le statut prestigieux des grandes productions M. G. M.), ces musiciens ont effectivement contribué à la mise en place d'un modèle musical "classique» dont les principes perdurent encore largement de nos jours 4 . Malgré l'idéal de la transparence professé par divers théoriciens (et les musiciens eux-mêmes ${ }^{5}$ ), la musique de film hollywoodienne s'impose fréquemment au premier plan sonore dès

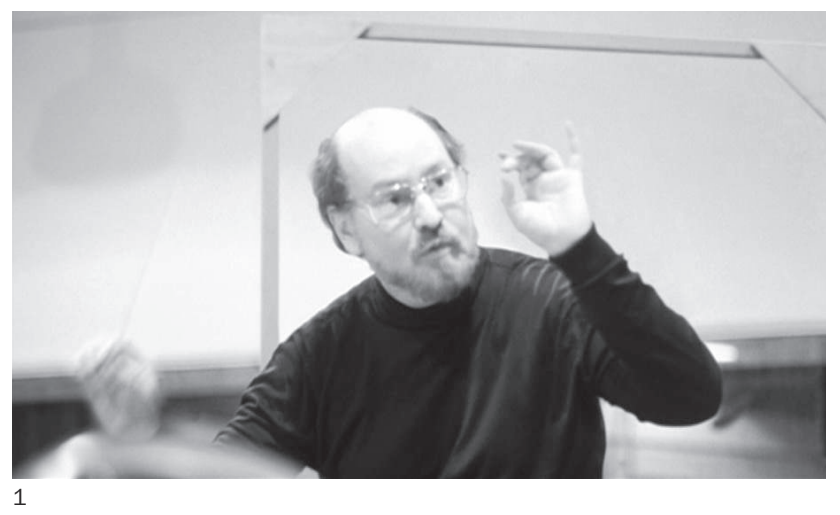

3 Voir mon article "L'esthétique wagnérienne et le cinéma", in Paul Lang (éd.), Richard Wagner. Visions d'Artistes. D'Auguste Renoir à Anselm Kiefer, Somogy / Musée d'Art et Histoire, Paris/ Genève, 2005, pp. 276-284, ainsi que "De 'nouveaux Wagner, pour l'écran: autour de l'émergence du grand spectacle cinématographique et musical ", à paraître dans un volume dirigé par Danièle Pistone (Université de Paris IV).

4 Voir Claudia Gorbman, Unheard Melodies. Narrative Film Music, Indiana University Press, Bloomington/Indianapolis, 1987; Kathryn Kalynak, Settling the Score: Music and the Classical Hollywood Film, University of Wisconsin Press, Madison \& London, 1992, Michel Chion, La Musique au cinéma, Fayard, Paris, 1995, pp. 113128; Pierre Berthomieu, La Musique de film, Klincksieck, Paris, pp. 21-75.

5 Voir les écrits de Kurt London, Maurice Jaubert, Herbert Stothart ou, dans une perspective critique, Hanns Eisler, cités dans mon article "De nouveaux Wagner...", op. cit. Sur l'adhésion des musiciens hollywoodiens à ce dogme de l'"inaudibilité", lire "Unheard Melodies? A Critique of Psychoanalytic Theories of Film Music ", in David Bordwell et Noel Carroll (éd.), Post-Theory: Reconstructing Film Studies, University of Wisconsin Press, Madison, 1996, pp. 230-247. 
6 R. Wagner, L'CEuvre d'art de l'avenir, Delagrave, Paris, 1928 [rééd.: Les Introuvables, Paris, 1976].

7 Cité par Roy Pickard, The Hollywood Studios, Muller, Londres, 1978, p. 140.

$8 \mathrm{~J}$. Bourgeois, "Musique dramatique et cinéma", Revue du cinéma, n 10, février 1948, pp. 25-33. Bourgeois est à la fois critique de cinéma et musicographe, auteur notamment d'un Richard Wagner, Plon, Paris, 1959.

9 «Par l'exemple de Williams, le son épique établi dans les thirties devint à nouveau un choix viable pour les compositeurs du cinéma hollywoodien contemporain. En fait, le score de L'Empire contre-attaque est un exemple magistral de la stabilité et de la puissance du modèle structurel qui sous-tend le score classique et la force de l'idiome post-romantique qui le guide." K. Kalinak, op. cit., pp. 188-189. que s'estompe la toute-puissance du verbal pour transmettre une forte charge émotionnelle et expressive ou pour souligner rythmiquement les actions visuelles (l'effet de mickeymousing s'applique non seulement aux dessins animés, mais aussi aux comédies ou aux films d'action). Cette appréhension à la fois spectaculaire et narrative de la musique de film s'inscrit dans le prolongement de préoccupations faisant elles-mêmes écho aux réflexions de Richard Wagner sur le Gesamtkunstwerk, perçu comme la grande synthèse du drame et de la musique. Même en réduisant le mythe à ses traits essentiels pour permettre à l'orchestre d'étendre ses possibilités polyphoniques, l'auteur de L'Euvre d'art de l'avenir (1849) assigne aux sonorités musicales un rôle qui consiste à fusionner avec le récit afin d'amplifier ce qui ne peut être dit ou montré. La musique doit à son sens servir les objectifs du poète et inféoder sa structure globale aux nécessités de l'action dramatique ${ }^{6}$. Cette mise en relation a été très vite reconnue d'une manière explicite, au sein même des studios californiens (la célèbre injonction de Sam Goldwyn aux musiciens de film : "Write Music like Wagner, only louder»!7) comme dans la réception européenne de la production hollywoodienne. Ainsi le critique Jacques Bourgeois estime-t-il, au sortir de la Seconde Guerre mondiale, que le cinéma américain a "recueilli l'héritage esthétique» du grand compositeur, "réinvent[ant] aujourd'hui peu à peu les procédés dramatiques wagnériens». La vision d'Objective Burma (1944, musique de Waxman) soulève son enthousiasme: "Qui voudrait aujourd'hui analyser mesure par mesure la Tétralogie pour la comparer avec telle partition réussie de film d'action [...] serait sans doute étonné de découvrir que la musique de Wagner est pour ainsi dire écrite pour le cinéma»8.

Si l'historiographie de la musique de cinéma s'accorde à percevoir un déclin relatif de ce type d'accompagnement symphonique au cours des années 1950-1960 au profit d'airs jazz ou pop, elle identifie généralement dans le travail de John Williams pour Un nouvel espoir (1977) le signal majeur d'une certaine résurgence de la musique orchestrale dans le contexte des productions de proue de l'industrie filmique. Les sonorités postromantiques composées par John Williams pour les blockbusters conçus par Steven Spielberg ou George Lucas ont imposé depuis trente ans une esthétique très influente au sein de l'industrie hollywoodienne, dont les productions se situent à la croisée d'un souci d'innovation technologique (le recours massif aux effets spéciaux) et d'un retour aux conventions spectaculaires propres à d'anciennes formes de divertissement promues par la culture de masse au moins depuis l'entre-deux-guerres 9 .

Il existe à l'évidence des degrés divers dans cette reprise de schémas traditionnels: si la puissance et l'ampleur émotionnelle dégagées par 
l'orchestre symphonique sont devenues effectivement incontournables, s'il est impossible de remettre en question la domination continuelle des principes fondamentaux du modèle «classique» orientés vers le renforcement des enjeux narratifs et la célébration du pathos, il faut par contre reconnaître que le recours au leitmotiv n'occupe pas une place centrale dans la plupart des scores hollywoodiens contemporains. Le procédé intervient avant tout dans le cadre d'œuvres centrées sur des univers narratifs complexes, épousant plus ou moins directement l'ambition mythologique de l'opéra wagnérien. Le meilleur exemple récent se situe dans la trilogie du Seigneur des Anneaux (réalisée par P. Jackson d'après le cycle littéraire de Tolkien, musique d'Howard Shore, 20012003), dont la quête d'une dimension opératique, explicitement revendiquée dans les bonus des éditions DVD, s'appuie sur le développement d'une série de motifs mélodiques étroitement liés à des éléments narratifs (personnages, lieux, valeurs, sentiments...) et tranche singulièrement avec les dernières tendances musicales du blockbuster qui, depuis une dizaine d'années, opèrent essentiellement à partir de figures répétitives empruntées au minimalisme de Phil Glass ou Steve Reich, de riffs percutants, d'accords martelés et de l'interaction entre le son symphonique et les rythmes électroniques (voir, à divers titres, le travail de Hans Zimmer, de Graeme Revell ou encore celui de Don Davis pour les Matrix entre 1999 et 2003).

Malgré la ressemblance flagrante entre certaines mélodies de John Williams et celles signées par Erich Wolfgang Korngold, le compositeur le plus éminent du cinéma classique hollywoodien (son «Main Title»10 de King's Row se retrouve par exemple dans les thèmes principaux de Star Wars et Superman) $\mathbf{1 1}$ et en dépit du recours dans Star Wars à des effets orchestraux évoquant aussi bien l'emphase sentimentaliste que le brio clinquant des films d'aventures tournés dans les années 1930-1940, ce rapprochement s'avère en fait moins pertinent en termes directement stylistiques que sur le plan des techniques employées pour interagir avec la dynamique du récit cinématographique. Ainsi le "Main Title» de Star Wars reproduit-t-il scrupuleusement la structure caractéristique des génériques de Korngold (voir par exemple The Sea Hawk ou Anthony Adverse) 12, à savoir l'alternance de deux motifs destinés à être repris et développés dans la suite de l'œuvre: tout d'abord une fanfare tonitruante aux cuivres, solidement ancrée dans les tons fondamentaux des accords via la succession d'intervalles de quarte ou de quinte ascendants, connotant la dimension hérö̈que de l'action virile; puis une ample mélodie pathétique mettant à l'honneur les cordes pour dévoiler la facette plus intime de ce même univers de bravoure, celle des grands sentiments
$10 \mathrm{Je}$ cite sous cette forme les titres attribués aux morceaux dans les éditions discographiques.

11 King's Row (Sam Wood, 1942); Superman (Richard Donner, 1978).

12 Anthony Adverse (M. LeRoy, 1936); L'Aigle des mers (The Sea Hawk, Michael Curtiz, 1940). 
13 Outre les textes des pochettes des éditions CD intégrales des bandes originales (RCA Victor / BMG Classics, 1997), qui rappellent par leur souci d'exhaustivité les commentaires des livrets réalisés pour les opéras de Wagner, de nombreux internautes ont abordé ces différents thèmes de manière très détaillée (du guide pratique "Star Wars music" sur Wikipedia aux analyses très érudites des films de la seconde trilogie par certains contributeurs du site jwfan.com, tels John Takis ou King Mark). romantiques. Tout comme Korngold, soucieux d'adapter au cinéma les critères de la musique "appliquée» pour la scène, Williams détermine une série de thèmes importants renvoyant aux protagonistes, aux espaces, voire aux valeurs propres à l'univers représenté : à côté du célébrissime "Main Title» associé à la Rébellion et à Luke, ainsi que l'«Imperial March" dédiée à l'Empire et à Darth Vader, on trouve notamment des motifs pour Obi-Wan / La Force, Leia, l'Empereur, la Rébellion, l'Etoile Noire, les Jawas, les Ewoks, Boba Fett, Lando Calrissian, Jabba, Anakin, Shmi, Qui Gon Jin, Darth Maul, les droïdes ou encore le général Grievous, sans parler de thèmes destinés à exprimer la nature passionnelle des relations amoureuses (Leia et Han Solo dans la première trilogie; "Across the Stars» utilisé dans la seconde pour souligner la passion entre Padmé et Anakin) ou encore des tensions importantes (deux thèmes récents qui cèdent à l'attraction des ostinati et des chœurs pseudo-médiévaux à la Carl Orff: "Duel of the Fates» pour la lutte entre les deux côtés de la Force; "Battle of the Heroes» pour la confrontation finale entre ObiWan et Anakin)13. Toujours dans la tradition de l'opéra wagnérien (et de l'accompagnement musical des films, au moins depuis l'"Age d'or» hollywoodien), Williams réutilise, reformule, adapte ses leitmotive au fil des différents épisodes de la saga, en fonction des nécessités narratives. Ainsi l'«Imperial March» abandonne-t-elle ses accents martiaux caractéristiques, exposés dès L’Empire contre-attaque, pour la mort rédemptrice de Darth Vader à la fin du Retour du Jedi, ou, dans la seconde trilogie qui nous projette dans le passé républicain, pour ses brèves associations au personnage d'Anakin enfant et adolescent, avant qu'elle ne retrouve dans La Revanche des Sith toute sa solennité, soulignant dès lors le basculement de ce protagoniste vers le côté obscur de la Force. Le thème de la Force fait pour sa part l'objet de réadaptations permanentes dans ce dernier film: il est exposé dans une version guerrière lors de l'ouverture, accompagne les péripéties d'Obi-Wan, apparaît en fond durant les dialogues dédiés à l'avenir des Jedi avant d'éclater durant le duel final sur Mustafar. Enfin, dans le même ordre d'idées et toujours dans La Revanche des Sith, "Across the Stars" se fait plus tourmenté lors des cauchemars d'Anakin prédisant la mort de Padmé. Par ailleurs, les possibilités de combinaison, d'enchevêtrement des thèmes sont constamment explorées, la partition glissant très rapidement d'une mélodie à l'autre en suivant le développement du récit visuel. Ainsi une séquence chez Lando Calrissian à la fin de L'Empire contre-attaque combine-t-elle en quelques minutes, outre le thème assigné au propriétaire des lieux, ceux de Luke, Yoda et la Force. 


\section{Thèmes musicaux et mythiques}

Dans l'idéal de Wagner, soucieux d'atteindre une portée d'ordre mythique, la relation intime entre le leitmotiv et son référent doit se révéler forte au point de s'apparenter à une forme de baptême fondamental où la dénomination musicale finirait par renfermer l'essence de l'élément narratif qu'elle est censée figurer. Claude Lévi-Strauss a montré les liens privilégiés existant entre mythe et musique, deux "langages qui transcendent, chacun à sa manière, le plan du langage articulé» et a rappelé à cet égard le rôle primordial de Wagner, ce «père irrécusable de l'analyse structurale des mythes" qui a ouvert la perspective selon laquelle "la structure des mythes se dévoile au moyen d'une partition»14. L'œuvre du compositeur est portée par l'ambition ésotérique de renouer avec les fonctions sociales et morales de l'art, telle qu'elle avait pu s'incarner au travers de la tragédie antique. Le fantasme du Gesamtkunstwerk est avant tout gouverné par le projet d'une reformulation moderne de cette dernière à partir de certains principes (la synthèse rythmique des modes d'expression; l'alliance entre dépouillement et puissance monumentale) 15. Par contre, Wagner choisit de substituer l'univers mythologique antique à une nouvelle base poétique inspirée de légendes populaires nordiques et germaniques, afin de mieux toucher, par cette reprise de racines culturelles communes, l'inconscient collectif des spectateurs contemporains et, partant, de (re)constituer ces derniers en tant que groupe social homogène, c'est-à-dire comme un "peuple»: il «transporte l'imagination dans les âges reculés et remue les fibres intimes de l'homme moderne»(Edouard Schuré) 16. Cette relecture des mythes s'appuie essentiellement sur l'exploitation de conventions nouvelles, qui ne visent à conserver de l'intrigue psychologique qu'une série de mouvements fondamentaux, exaltant les valeurs et les sentiments essentiels, soulignant les enjeux philosophiques et les conflits de forces sous-jacents au récit. Comme le rappelle René Dumesnil, il est nécessaire que le drame "passe de l'analyse à la synthèse, que la vérité qu'il contient soit assez large pour avoir une valeur universelle. [...] Ses personnages seront eux-mêmes des symboles vivants »17.

C'est notamment cette tâche qui est attribuée au leitmotiv dans le drame musical scénique, puis dans ses avatars filmiques. En témoignent de nombreuses œuvres du cinéma classique hollywoodien, qui exploitent les variations (évocations in absentia, révélation progressive d'une identité à l'extérieur comme au sein même de la diégèse) rendues possibles par la fusion, souvent scellée dès le générique, entre un thème musical et le centre identificatoire du récit, qu'il s'agisse d'un protagoniste (Laura, The Informer) ou du noud psychologique fondamental sur
14 Claude Lévi-Strauss, Mythologiques. Le Cru et le Cuit, Plon, Paris, 1964, pp. 23-24.

15 Edouard Schuré, Le Drame musical. Richard Wagner. Son œuvre et son idée, Librairie Académique Perrin et Cie, Paris, 1928 [20éd.], p. 273.

16 ld., pp. 286-287.

17 R. Dumesnil, Richard Wagner, Les Editions Rider, Paris, 1929, p. 38. 
18 Laura (Otto Preminger, 1944); Le Mouchard (The Informer, John Ford, 1935); Casablanca (Michael Curtiz, 1943); L'Ombre d'un doute (Shadow of a Doubt, Alfred Hitchcock, 1943).

$19 \mathrm{H}$. Eisler, Composing for the Films, Oxford University Press, New York, 1947, pp. 5-6. II fustige de manière plus générale l'idéal de la fusion synesthésique (y compris la collaboration Eisenstein-Prokofiev pour Alexandre Nevsky, en 1938) comme une "erreur" commise sous l'uinfluence des conceptions wagnériennes du Gesamtkunstwerk" (Id., pp. 77-78).

20 II souligne à ce sujet la "formidable prédominance de l'élément germanique, même et surtout en Amérique, dans la corporation des musiciens de films" (M. Jaubert, "Les Arts", Esprit, avril 1936, p. 117).

21 Voir à ce sujet les commentaires de Philippe Lacoue-Labarthe, Musica Ficta (Figures de Wagner), Christian Bourgeois, Paris, 1991, pp. 219-221.

22 T. W. Adorno, Essai sur Wagner, Gallimard, Paris, 1966 [1962], en particulier pp. 121-122, 133 et 139. Voir également Le Caractère fétiche dans la musique, Allia, Paris, 2001 [1938; 1973], p. 35. lequel se structure le développement du récit («As Time Goes By» pour la romance de Casablanca; "The Merry Widow» pour le mystère criminel de Shadow of a Doubt) 18. Quelquefois, le baptême musical redouble celui qui s'opère dans le cadre de l'univers diégétique: dans Anthony Adverse, Korngold élabore le leitmotiv du personnage principal en s'inspirant du rythme du dialogue prononcé lors de la séquence où on lui attribue un nom pour la première fois. De même, dans La Revanche des Sith, le thème musical de Darth Vader apparaît conjointement à la parole performative de l'Empereur qui s'adresse à Anakin: "You shall be known as Darth Vader». A la fin du film, les naissances de Luke et Leia, et la mention de leurs prénoms respectifs sont également soulignées musicalement par la brève citation de leurs leitmotive.

Un débat s'est ouvert depuis longtemps autour du schématisme avec lequel la musique de film, y compris la plus sophistiquée, peine à atteindre cette dimension élevée du mythe. Si certains fustigent l'usage schématique, voire mécanique du leitmotiv au cinéma (le compositeur Hanns Eisler le voit par exemple réduit «au niveau d'un laquais musical, qui annonce son maitre»19), d'autres perçoivent directement chez Wagner lui-même la source d'une simplification à outrance du discours musical en fonction d'impératifs narratifs. Ainsi le compositeur Maurice Jaubert stigmatise-t-il, en 1936 déjà, l'usage hollywoodien des «moins recommandables des recettes wagnériennes» $\mathbf{2 0}$, un reproche que formulera également Theodor Adorno à l'encontre de la "fantasmagorie» wagnérienne. En effet, celle-ci lui semble préfigurer en grande partie la réification de l'art musical opérée par la culture de masse au $\mathrm{XX}^{\mathrm{e}}$ siècle, l'auteur du Ring empruntant à son sens le détour mythologique pour masquer la déformation brutale et radicalement nouvelle impliquée autant par l'inféodation du langage musical au grand spectacle scénique que par une forme de saturation musicale qui déjoue en fin de compte les propres principes du compositeur 21. La restauration du "grand art» rêvée par Wagner aurait dès lors abouti à une juxtaposition excessive d'effets hyperboliques et stériles. Pour qualifier tout son dégoût face au caractère «suiviste» de la composition wagnérienne, le philosophe allemand emploie une formule anachronique tout à fait révélatrice pour notre propos, puisqu'il qualifie la «facture musicale wagnérienne» de "proprement cinématographique»22. Le film devient, à rebours, le modèle même des tendances qui le préfiguraient au sein de l'opéra, un demi-siècle auparavant.

Par certains traits stylistiques et connotations culturelles, les thèmes musicaux les plus importants de Star Wars renvoient effectivement aux tensions fondamentales qui sous-tendent cet univers particulier. Cette 
démarche s'appuie tout d'abord sur la mise en avant du caractère traditionnel, voire passéiste de l'esthétique musicale. D’après Williams lui-même, le choix de sonorités à la fois enjouées et martiales pour les thèmes les plus connus de Star Wars procède de la volonté de soulever "quelque souvenir de Buck Rogers ou du Roi Arthur ou quelque chose de plus ancien encore dans le fond de nos esprits [...], les mémoires de vies vécues dans le passé [...] [Cette musique] possède cette sorte de résonance - elle fait résonner au sein de nous-mêmes l'ancienne vie héroïque que nous avons tous vécue. »23

Si les deux mélodies centrales de Star Wars ("Main Title» et «Imperial March») partagent les mêmes sonorités martiales, via des arrangements en triolets qui dynamisent singulièrement les contours très élémentaires de ces thèmes, elles proposent une nette différenciation musicale des deux forces antagonistes de l'univers représenté. Le Main Title, d'abord fanfare enjouée, puis hymne au lyrisme triomphant, exprime à merveille la militance réactionnaire des rebelles pour la résurgence d'un passé harmonieux et utopique, où la technologie doit absolument demeurer au service de l'être humain. Au contraire, les musiques liées à l'Empire ou au Côté obscur signalent toute l'artificialité d'un monde sinistre dominé par les machines, leur répétitivité morbide reposant sur des harmonies qui, sans être dissonantes - les expériences véritablement atonales sont réservées aux séquences de bataille, ou à certaines rencontres monstrueuses (voir infra) -, rompent avec les développements en cadences tonales. Tout comme le thème de l'Empereur ou celui de l'Etoile Noire, qui se caractérisent par la juxtaposition de blocs harmoniques disjoints, la base rythmique de l'«Imperial March» oscille en effet entre le ton fondamental (un sol à l'unisson, sur trois temps) et un accord en position de sixte mineure (un accord de Mi bémol mineur sur le quatrième temps de la mesure). Cette indétermination harmonique, créée par la simplicité du matériau, s'accentue avec la mélodie de cette même marche: une triade d'apparence majeure (Mi bémol), mais dont l'insistance sur la tierce (le sol déjà martelé en basse) donne le sentiment d'une tonalité en Sol mineur 24. La dualité du personnage d'Anakin/Vader, tiraillé entre les deux pôles de l'humanité et de la machine, est en quelque sorte posée d'entrée de jeu grâce à l'ambiguïté que suggère le thème de l'«Imperial March".

Quoi qu'il en soit, les accords de Mi bémol majeur et de Sol mineur sont l'un et l'autre très proches du Si bémol majeur du Main Title, sur lequel s'ouvre chaque épisode de la saga, comme pour rappeler le caractère primordial de cet accord, son statut de tonalité fondatrice d'où émerge l'univers tout entier de la série. A chaque "générique",
23 Entretien avec Craig L. Byrd, in FilmScoreMonthly, janvier 1997.

24 Pour une analyse plus fouillée de ces mélodies, voir K. Kalinak, op. cit., pp. 194-197 et James Buhler, "Star Wars, Music, and Myth", in James Buhler, Caryl Flinn et David Neumeyer (éd.), Music and Cinema, Wesleyan University Press, Hanovre/Londres, pp. 33-35. 
25 James Buhler montre dans son analyse que le film est structuré par une tension entre ces deux pôles de la musique/nature et de l'effet sonore/technologie, le mixage accordant souvent une prépondérance de l'un sur l'autre en fonction de la tension entre les deux côtés de la Force.

26 Voir mon article "Un nouveau règne de la terreur . La voix de L'Homme invisible et les mythes de la dictature radiophonique", in Laurent Guido (éd.), Les Peurs de Hollywood, Antipodes, Lausanne, 2006, pp. 59-81. l'hymne de la 20th Century Fox, qui accompagne le logo de la firme hollywoodienne, cède sa place à un silence sur fond de l'espace infini et à l'évocation du passé comme de l'ailleurs mythiques dans lesquels se situe le récit ("A long time ago, in a galaxy far, far away...»). C'est alors que surgissent simultanément le titre lumineux et l'accord initial du thème principal en Si bémol majeur. Ce synchronisme absolu entre musique et image, qui insiste sur le caractère élémentaire des deux aspects qui coïncident (la note de basse est répétée plusieurs fois avant que ne débute la mélodie), fonctionne alors comme un moment baptismal pour la cosmologie d'une œuvre orientée vers le désir nostalgique d'une fusion régressive avec un archaïsme diffus. La disparition du titre dans la profondeur du cadre signale le caractère précieux de cette plénitude originale. Elle provoque à la fois le sentiment de perte de cette harmonie originelle - et le désir corrélatif de la retrouver ainsi que celui de la profondeur, lui-même renforcé sur le plan musical par le développement de la mélodie. Ce discours sonore déploie avec détermination et précision les notes de l'accord de Si bémol majeur, attaquées en une succession d'intervalles ascendants (quatre, quinte, puis septième mineure) avec une instrumentation de fanfare (cuivres, cymbales...).

C'est à partir de cette donnée fondamentale et une sorte de conclusion ouverte sur la neuvième de la tonalité de base, puis la dissolution de celle-ci, que s'engage généralement la rupture introduite par la guerre technologique, immanquablement signalée sur le plan sonore par le surgissement d'effets de design acoustique liés aux éléments mécaniques (moyens de transport, droïdes, etc.) 25 Tout comme dans l'opéra wagnérien, où l'anneau peut servir le bien comme le mal en fonction de son possesseur, et conformément à une tradition de représentation américaine solidement engagée dans le cinéma des années 1930-194026, la technologie n'est pas condamnée en soi dans Star Wars, mais dans ses dérives et ses appropriations anti-humanistes. Plus nuancé que la charge tennysonienne du Seigneur des anneaux de Tolkien (une diatribe qui devient totalement schizophrénique dans ses récentes adaptations cinématographiques), le discours de Star Wars sur l'univers mécanique se résume en fin de compte à la trajectoire d'Anakin, dont la fascination pour les machines, entamée sous l'angle de la maîtrise (via l'iconographie populiste du pilote-mécanicien de génie), finit par révéler ses tendances excessives (la perte d'autonomie identitaire du héros par sa transformation partielle en machine). A l'évidence, une contradiction se fait jour entre le discours critique que développe Star Wars, en particulier dans la seconde trilogie, sur les dangers totalitaires de l'outrance technique 
(et économique), et le statut emblématique qu'occupe aujourd'hui cette œuvre dans l'industrie mondialisée du divertissement, fondée sur l'hybridation digitale de l'humain et de la machine et sur la mise en réseau des circuits d'information et de communication. Dès ses débuts, cette série de films a basé son succès sur un rapport fétichiste au spectacle de la technologie, accordant une large place au déploiement des objets mécaniques, en particulier par l'entremise d'effets sonores censés refléter les bruits produits par cet univers machinique.

\section{L'ancien et le nouveau}

A la fin des années 1970, le succès international rencontré par Star Wars participe certes d'une importante mutation industrielle et économique, centrée autour du blockbuster et qui ne cessera de s'amplifier au cours des trois décennies suivantes, en phase avec certains progrès liés notamment à l'émergence des techniques numériques dans le secteur audiovisuel. C'est pourtant une démarche essentiellement réactionnaire que recouvre Star Wars, non seulement parce que cette série filmique signale le retour au premier plan de l'industrie hollywoodienne après le déclin relatif de l'après-guerre, mais aussi en raison de ses choix esthétiques qui réduisent systématiquement la portée révolutionnaire des nouvelles technologies pour les mettre au service d'anciennes idées, conformément à une certaine logique régressive et nostalgique propre à la période dite "postmoderne» étudiée par Jean Baudrillard ou Fredric Jameson. En re-dynamisant par les effets spéciaux et le son surround les formules postwagnériennes du grand spectacle hollywoodien "classique», les films de Star Wars tentent avant tout de porter à un niveau épique et mythologique l'univers de l'industrie culturelle.

Pour son cycle de (space) opéras, Lucas a donc procédé à un travail comparable à celui de Wagner, en croisant diverses sources narratives, puisant notamment dans de grandes références philosophiques, mythologiques, anthropologiques ou religieuses. C'est le plus souvent sur la base de ces influences que l'on a tenté de légitimer l'objet Star Wars sur le plan culturel, en s'appuyant surtout sur la relation privilégiée nouée par Lucas avec le spécialiste des mythes héroïques Joseph Campbell (Les Héros sont éternels / The Hero with a Thousand Faces, 1949). Il me paraît pourtant ici moins intéressant de rapporter l'œuvre de George Lucas à ces grandes idées ou récits légendaires (ainsi la trajectoire d'Anakin apparaît-elle aisément comme une relecture de Prométhée ou de Faust), que d'inscrire au contraire Star Wars dans sa relation à la culture de masse du $\mathrm{XX}^{\mathrm{e}}$ siècle, en laissant de côté la question pourtant cruciale, mais hors de propos ici, du contexte socio-politique ${ }^{\mathbf{2 7}}$.
27 Contrairement au refus de l'histoire inhérent à l'entreprise de Lucas (à l'ailleurs mythique évoqué dans les films fait écho un mode de production non linéaire), il faut bien considérer ces films comme des œuvres de leur époque, d'une part au plan des stratégies industrielles - le démarrage de la nouvelle trilogie adopte les canons du divertissement pour enfants qui imprégnaient déjà de nombreux passages du Retour du Jedi-; d'autre part au plan des allusions au contexte socio-politique (voir mon introduction au recueil Les Peurs de Hollywood, op. cit., p. 38). Cette dimension se situait d'ailleurs également au cœur de l'opéra wagnérien, qui se référait notamment à l'industrialisation en cours dans la deuxième moitié du $\mathrm{XX}$ siècle. Par ailleurs, les stéréotypes raciaux qui émaillent la saga (en particulier dans La Menace fantôme) participent d'un même rapport aux représentations sociales que l'antisémitisme de Richard Wagner, perceptible par exemple dans sa représentation des Nibelungen. 
28 Robert Benayoun et Michel Ciment, "Entretien avec George Lucas ", in Positif, n 197, septembre 1977, p. 59.

29 Entretien avec Craig L. Byrd in FilmScoreMonthly, janvier 1997, www.filmscoremonthly. com/features/williams.asp); entretien avec David Thomas in Total Film Magazine, $\mathrm{n}^{\circ} 8$, septembre 1997, pp. 74-79.

30 Entretien pour la station NPR, repris sur le site www.jwfan.com en mai 2005.

31 Entretien avec Phillip Huscher ["Sound and Image: John Williams at the Chicago Symphony"], sur www.cso.org/main.taf

32 Entretien avec Craig L. Byrd, op. cit.

33 D. Schroeder, op. cit., p. 215.
D'emblée, le discours même des concepteurs d'Un nouvel espoir était essentiellement guidé par le projet de recréer les conditions du grand spectacle de divertissement tel qu'il avait notamment été conçu au sein du cinéma classique hollywoodien, en adaptant ses principes (celui du film historique ou d'aventures épique) à d'autres éléments liés à la culture des adolescents américains de l'après-guerre, perçue avec une nostalgie régressive après les bouleversements sociaux des années 19601970. Si Lucas admet en 1977 avoir "voulu réaliser la fiction hollywoodienne classique à l'ancienne que les gosses adorent»28, John Williams lui emboîte le pas dans différents entretiens où il souligne à son tour l'absence de prétentions esthétiques de son travail sur Star Wars. Il rappelle en outre sa prise de conscience tardive de la dimension "pseudomythologique» de l'œuvre, grâce aux analyses de la saga proposées par Joseph Campbell. De celles-ci, il retient essentiellement une conception romantique de l'art musical en tant que langage universel par excellence, capable de susciter la nostalgie d'anciens liens communautaires: "Nous ne connaissons pas le futur, mais nous partageons certainement un passé glorieux. Nous nous en souvenons dans le langage et dans le pré-langage. C'est là où la musique se situe, c'est dans cette région de nos âmes qu'elle peut s'exprimer. "29 Refusant absolument toute comparaison avec Wagner ${ }^{30}$, Williams insiste avant tout sur le "fun» provoqué par l'exploitation des clichés orchestraux liés au film d'action et d'aventures, y percevant même la possibilité pour le London Symphony Orchestra (fig. 2) de quitter un moment l'espace de la «musique sérieuse»31. Vingt ans après Un nouvel espoir, le compositeur avoue encore son étonnement devant le succès international remporté par ce qu'il considérait au moment de sa réalisation comme "un film du samedi après-midi pour les enfants, une sorte de pop-corn, une émission de Buck Rogers, un bon sound and light show pour les jeunes»32.

Ce dernier aspect a notamment frappé l'attention de David Schroeder, d'après lequel Star Wars s'apparente avant tout au «Ring de Wagner, pour les adolescents", recyclant pêle-mêle, à côté d'emprunts à Eisenstein ou Kurosawa: The Wizard of $\mathrm{Oz}$, Tarzan, Robin des bois, le film d'horreur, les westerns, les aventures de pirates, les comics de superhéros et d'aventures spatiales à la Flash Gordon et Buck Rogers, la propagande cinématographique de la Seconde Guerre mondiale, en bref "une épopée wagnérienne avec un assortiment de créatures de dessins animés, de vilains méchants, de fusillades de jeux vidéo, de musique qui fait vibrer les cœurs...»33. C'est justement cette dimension qui constitue pour Robert Benayoun l'intérêt d'Un nouvel espoir, un tel film témoignant à son sens d'une "absence de recul volontaire par rapport aux mythes». Selon le 


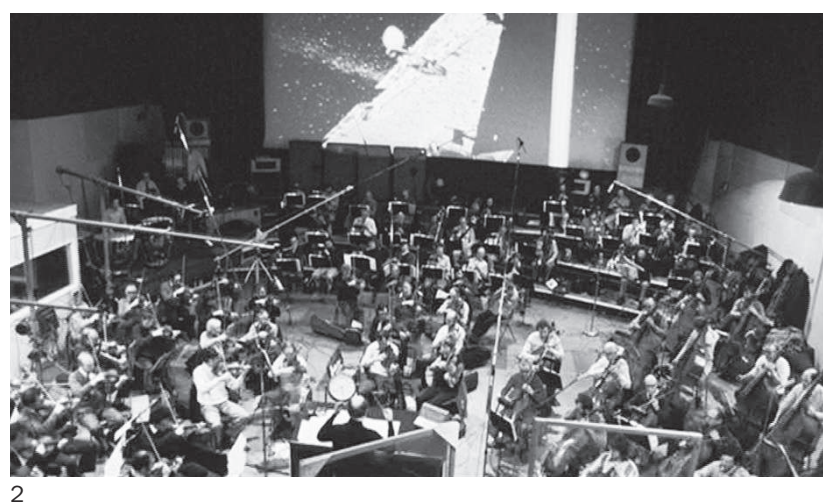

critique de Positif, la tension mythologique immédiate propre au cinéma de narration et d'action hollywoodien se teinte chez Lucas d'une nostalgie pour les modes de vie et de consommation des adolescents: "son odyssée de l'espace est un hamburger géant, un super ice-cream de péripéties mécaniques, un dragster trafiqué [...], un tutti-frutti de clichés cinéphiliques transcendés par son génie de mécano planant»34. En vertu d'une forme d'«intellectualité assez perverse», l'aspect feuilletonesque qu'identifie Benayoun (il cite des sérials muets comme Fantômas ou Fu $M a n c h u)$ serait comme "anobli sans cesse par la somptuosité des effets, la richesse du bestiaire, la désinvolture des événements montrés à l'écran et la frénésie du rythme général.» Cette méthode caractérise en effet la démarche esthétique de la saga tout entière, y compris dans les films de la seconde trilogie où s'accentue même un climat désuet d'action "à l'ancienne", entre poursuites rocambolesques et altercations "cape et épée" avec des villains grandiloquents, des chasseurs de primes ou des espions lovés dans l'ombre des colonnes, tout cela sur le tapis rythmique alerte fourni par les chatoiements pyrotechniques et la partition énergique de John Williams.

Cette collusion entre l'ancien et le nouveau au sein de Star Wars est constamment provoquée par l'emploi de la musique. Dans le volet initial, la fanfare d'Alfred Newman pour le carton inaugural de la 20th Century Fox provient d'ailleurs d'un ancien enregistrement de 1954, ce qui renforce l'inscription du film dans la continuité du cinéma classique hollywoodien. En phase avec les normes de cette dernière période, l'esthétique musicale de Williams provoque donc autant le sentiment d'un retour en arrière que celui d'une rupture de ton par rapport à l'horizon d'attente d'un film d'aventures spatiales. Comme le signale Lawrence E. MacDonald, "pour un public plus âgé, la musique richement orchestrée de Williams paraissait faire resurgir l'époque d'Erich Korngold,
34 Robert Benayoun, "L'Horizon retrouvé. ‘La Guerre des étoiles ' ", Positif, n 197, septembre 1977, pp. 46-50. 
35 Lawrence E. MacDonald, The Invisible Art of Film Music. A Comprehensive History, Ardsley House, New York, 1998, p. 227.

36 Entretien avec David Thomas, op. cit.

37 Entretien avec Phillip Huscher, op. cit. alors que pour des spectateurs plus jeunes, le son d'un grand orchestre constituait une vraie nouveauté»35.

\section{Drame musical ou musique dramatique?}

Chez Williams, la relation entre l'image et la musique ne se limite pas exclusivement à la dimension narrative: elle s'appuie fréquemment sur les mouvements visuels, les sinuosités formelles des séquences d'action. Evoquant l'autre pan essentiel du modèle musical «classique» - le soulignement rythmique de certains gestes ou trajectoires écraniques, également pratiqué intensément par Korngold ou Steiner -, Williams met souvent l'accent sur le dynamisme extraordinaire du mickeymousing. A propos de son score pour Le Monde perdu: Jurassic Park (The Lost World: Jurassic Park, S. Spielberg, 1997), il affirme par exemple que les balancements incessants liés aux déplacements des créatures de synthèse impliquent «un travail massif de symphonic cartooning. Vous devez faire coïncider exactement la musique aux tournoiements rythmiques des dinosaures et créer ces drôles de ballets» $\mathbf{3 6}$. Les séquences d'action les plus développées de la saga Star Wars (c'est-à-dire les grandes batailles qui jalonnent le récit, épisode après épisode, en particulier celles de Hoth dans L'Empire contre-attaque, d'Endor dans Le Retour du Jedi et de Géonosis dans L'Attaque des clones) témoignent de ce souci d'épouser musicalement les contours de l'action, en jouant des surprises et des changements continuels de rythme qu'implique un tel exercice. Si l'on accorde du crédit aux déclarations de Williams, sa méthode générale consiste à ne pas lire le scénario à l'avance, et à ne prendre contact avec le film qu'au moment où celui-ci est disponible dans un montage assez avancé pour que le musicien puisse se faire une idée assez précise du rythme du morceau à composer. Cette perception des «rythmes de montage" d'un film constitue donc une base essentielle de l'écriture musicale de Williams, autorisant la possibilité d'obtenir la «sensation du flux et reflux cinétique» ainsi que des ralentissements et accélérations, c'est-à-dire des endroits où l'on peut "capter les rythmes des films»37. Le tempo représente à son sens le problème essentiel de la composition pour le cinéma. Cette opinion peut surprendre de la part d'un musicien généralement cité en exemple d'une conception narrative de la musique. Au-delà des jeux de référence, d'extension et de développement entre leitmotive, Williams estime en effet ne pas avoir cherché à créer une véritable "unité architecturale» entre les différents épisodes de Stars Wars, chaque film ayant été selon lui abordé "comme une entité séparée». Le résultat final lui apparaît un "heureux accident», une "métamorphose naturelle mais inconsciente de thèmes musicaux qui 
semblent avoir plus d'interrelations architecturales [qu'il n'a] voulu y placer." La manière dont il relate son travail pour Un nouvel espoir relève bien de la démarche empiriste et rythmique décrite plus haut: "Je me souviens avoir vu le film et avoir réagi à ses atmosphères, ses énergies et ses rythmes. C'est toujours pour moi la meilleure manière de saisir un film - à partir de l'image visuelle elle-même et sans les préconceptions qui pourraient découler du scénario.»38 A l'instar des séquences de combat ou de poursuite qui scandent les films de Michael Curtiz mis en musique par Korngold (Les Aventures de Robin des Bois / The Adventures of Robin Hood en 1938; L'Aigle des mers / The Sea Hawk en 1940), c'est dans les passages d'actions mouvementées, où les informations visuelles se succèdent rapidement avec des rythmes souvent complexes, que peut se mesurer la virtuosité de l'écriture musicale. Chez Williams, des pièces presque dénuées de référence aux leitmotive peuvent atteindre des sommets de surprise et de dynamisme, le discours musical se détachant nettement de son ancrage postromantique pour emprunter aux techniques d'écriture plus contemporaine, à l'instar d'un Jerry Goldsmith. Ainsi la musique accompagnant la poursuite dans Coruscant au début de L'Attaque des clones élabore-t-elle un flux au relief sans cesse accidenté, où se succèdent ostinati frénétiques, changements brusques de volume, effets de polyrythmie et instrumentations originales (des percussions extraoccidentales aux sonorités growl des cuivres).

John Williams ne semble néanmoins pas partager le rêve, exprimé notamment par Korngold, de réaliser des morceaux musicaux qui puissent à la fois servir les intérêts narratifs d'un film et conserver leur autonomie en tant que pièces de concert. Rappelant la nature «journalistique » $\mathbf{3 9}$ du travail du compositeur de cinéma (en 1997, il indique qu'il écrit en moyenne un score en deux à trois mois), Williams épouse tout d'abord, sans grande nuance, le discours dominant de la transparence: la musique ne doit pas chercher à être perçue par les spectateurs, au-delà des reprises de petits motifs destinés à cimenter la cohérence de l'ensemble du film («memorability from reel to reel»)40. Il juge par conséquent "contre-productif» pour un compositeur de rechercher l'attention du public et estime même que cette optique tend le plus souvent à rendre la musique de film «banale et simpliste» $\mathbf{4 1}$. L'impact considérable de la partition musicale de Star Wars lui semble en fin de compte totalement dépendant de l'œuvre qu'elle accompagne, puisqu'elle est à son sens "guidée presque mesure par mesure par le film lui-même» $\mathbf{4 2}$.

La réception exceptionnelle de la musique de Star Wars lui donne pourtant tort: les thèmes principaux de la saga figurent parmi les mélodies les plus connues de la fin du $\mathrm{XX}^{\mathrm{e}}$ siècle, passées depuis longtemps
38 Entretien avec Craig L. Byrd, op. cit.

39 "Si le compositeur de cinéma imagine, comme le fait un compositeur de concert, que le public va donner $95 \%$ de son attention à la musique, il commet une grave erreur, parce que la musique de film doit être conçue pour être écoutée avec les effets sonores et le dialogue - nous devons admettre que nous écrivons tout le temps de l'accompagnement." Entretien avec Phillip Huscher, op. cit.

40 "Quelque chose du troisième acte, que vous pouvez saisir à nouveau et citer comme un vieil ami du premier acte, cela peut faire partie de la structure de ce qui rend la bande originale d'un film unifiée, solide et efficace." (Entretien avec David Thomas, op. cit.)

41 ld.

42 Entretien avec Phillip Huscher, op. cit. 
dans l'imaginaire collectif (des matches de baseball aux bandes de propagande d'Al Qaïda, en passant par les sonneries de téléphones portables). Plus spécifiquement, les sonorités musicales jouent dans Star Wars un rôle indispensable, apportant une dimension épique que les images ne parviennent pas toujours à produire par elles-mêmes. C'est l'argument de David Schroeder, selon lequel la magie technologique ne suffit pas à apporter l'ampleur dont manquent à son avis les personnages de la série: «la musique presque continuelle de Williams élève le niveau des séquences au fil de la projection, et donne l'impression qu'elles prennent une dimension monumentale [...] Essayez de regarder ces films sans le son et voyez quelle impression ils provoquent. Pas grand chose.»43 Cette critique paraît d'autant plus pertinente si l'on pense au démarrage de la seconde trilogie - et à de larges pans de l'ensemble de la série -, où Lucas peine à ancrer sa relecture de la culture populaire hors d'une systématisation infantile de l'anthropomorphisme cartoon (droïdes comme bestiaire). Inégale, cette pratique disqualifie fréquemment Star Wars sur le terrain de l'épique, même dans son versant le plus outrancier. L'écoute des pièces imposantes composées dans le style de Miklos Rozsa pour certaines séquences - par exemple le défilé précédant la course de pods dans La Menace fantôme, avec ses successions (récurrentes chez Williams) de modulations autour d'une mélodie lancinante à chaque fois relancée par l'intro en fanfare du "Main Title», ou le morceau accompagnant les joutes dans l'arène de L'Attaque des clones, qui déroule un tempo marcato en répartissant les mêmes figures rythmiques sur l'ensemble de l'instrumentation - semble effectivement renvoyer à des formes autrement plus intenses et spectaculaires que celles proposées par les images.

Ce sont certes les films eux-mêmes qui ont inspiré les compositions musicales. Mais, en produisant à partir de ce point de départ matériel une double stylisation (d'une part en capturant l'essence des connotations héroïques et mythiques que cherchent à produire les films, d'autre part en saisissant les lignes rythmiques essentielles de l'action visuelle), la musique devient absolument nécessaire à l'explicitation des enjeux et des sentiments fondamentaux, sur le double plan narratif et émotionnel. La grande complexité de la texture sonore de la seconde trilogie rend néanmoins cette efficacité musicale moins perceptible, en raison de la place importante accordée au vacarme sophistiqué produit par les vaisseaux, les armes, les droïdes... Contrairement à ce qu'affirme Williams lui-même, ces bruits de machines n'entrent jamais véritablement, comme pour les dialogues, dans un rapport symbiotique avec la musique (mixée souvent en background), mais tendent à constituer un discours parallèle à cette dernière (on rejoint la dichotomie vue plus 
haut entre nature et technologie, portée par ces deux matières de l'expression sonore au cinéma).

Star Wars ne s'arrêtant pas aux films, il faut encore considérer les supports musicaux proposés aux consommateurs : ainsi les CD comportant des extraits de la bande originale avant remontage et coupes, ou encore des versions de concert. Certaines éditions intégrales, disponibles pour les épisodes I et IV-VI, s'efforcent de placer les passages musicaux dans la continuité de l'action cinématographique, permettant ainsi de suivre l'avancée du récit sous une forme exclusivement musicale, un peu de la même manière que les mélomanes écoutent l'enregistrement d'un opéra. Ainsi, le DVD Star Wars. A Musical Journey $\mathbf{4 4}$ fourni en complément de la première édition discographique de La Revanche des Sith présente les principales mélodies de Star Wars sous la forme de courts métrages qui retracent les étapes essentielles de la saga, le montage des images obéissant cette fois au développement du discours musical.

\section{A Musical Journey, vers une synthèse musicale et visuelle}

L'enchaînement des seize segments de ce "voyage musical» d'une heure peut être visionné avec ou sans les introductions où l'acteur écossais Ian McDiarmid 45, sobrement cadré en plan rapproché dans les studios d'enregistrement londoniens d'Abbey Road 46, s'adresse à la caméra pour rappeler très succinctement quelques données nécessaires à la compréhension narrative de chaque clip (avec cette option, la durée du bonus s'allonge de dix minutes supplémentaires) (fig. 3). Conformément à une logique d'attraction spectaculaire proche de celle des bandes-annonces, mais sur un mode plus synthétique et démonstratif, chacun de ces courts métrages résulte d'un montage rapide de plans extraits des six films de Star Wars. Leur objectif principal est de rendre compte de l'un des tournants importants du récit, qu'il se rapporte à un événement marquant ou à un personnage particulier. La progression des parties obéit à l'évolution chronologique des six films: on nous conduit de la conspiration du Côté obscur à la victoire finale de la Rébellion ou encore de l'émergence d'Anakin à sa rédemption, en insistant avec plus de détails sur les séquences de la première trilogie qui est déjà évoquée à partir du cinquième clip. Dans la plupart des segments, ce mouvement d'ensemble s'accompagne néanmoins d'une autre démarche. Celle-ci consiste à circuler au sein de différents épisodes de la saga pour établir des liens entre des protagonistes ou des situations similaires. Ainsi, les batailles finales de La Menace fantôme et de L'Attaque des clones se développent parallèlement à la grande confrontation avec Darth Maul, qui scande l'évocation plus générale des conflits apparaissant à la fin
44 Star Wars. A Musical Journey (2005), une production Lucasfilm Ltd. et Sony Classical réalisée par Tippy Bushkin, écrite par Jamie Richardson et montée par Jeremy Stuart.

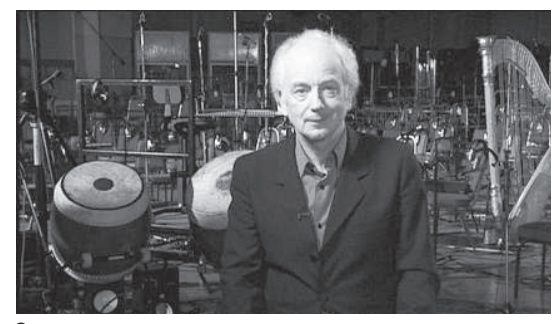

3

45 L'interprète de Palpatine est ici investi d'un rôle de conteur qui n'est pas éloigné de la fonction de "maître du jeu" qu'occupe le personnage qu'il incarne au sein de la série filmique.

46 Par cette mise en scène de l'espace de production (dans l'arrière-plan apparaissent même des instruments et les sièges vides de l'orchestre, soit-disant avant que ne débute l'enregistrement des thèmes de La Revanche des Sith), on suggère donc que la musique symphonique aux accents nostalgiques de Williams participe elle aussi du passé noble, prestigieux et légitimé que Star Wars assimile à l'ancrage britannique. C'est dans cette région européenne, d'où sont partis les pères fondateurs des Etats-Unis, que l'on a d'ailleurs recruté de nombreux interprètes des membres élitaires de la République et de l'Empire (Alec Guinness, Peter Cushing, Ian McDiarmid, Ewan McGregor, Liam Neeson, Terence Stamp, Christopher Lee...). 
47 C'est encore le cas avec A New Day Dawns (sur "Throne Room/Finale" tiré d'Un nouvel espoir) qui souligne la parenté des célébrations finales des épisodes I, IV et VI.
Glissement de la production à la fiction: dans A Musical Journey, George Lucas et John Williams sont isolés sur le fond noir ouvrant sur l'univers de Star Wars.

de l'ère républicaine (Dark Forces Conspire, basé sur le morceau "Duel of the Fates" tiré de La Menace fantôme). De même, A Sanctuary Moon associe la bataille d'Endor à celle de Naboo en raison de l'affrontement identique que se livrent forces mécaniques et "tribales", les mouvements burlesques des Gungans s'adaptant bien à une musique de mickeymousing originellement adaptée aux déplacements presque enfantins des Ewoks (pièce "The Forest Battle»). A l'instar de A Defender Emerges, portrait plurivoque de la princesse Leia à partir d'un développement de son thème musical, An Empire is Forged (évidemment monté sur "The Imperial March») renvoie pour sa part à l'évocation plus spécifique du personnage de Darth Vader, de sa puissance et de sa cruauté démesurées, ainsi que de son rapport à Luke. Si A Fateful Love (utilisant "Across the Stars») et A Bond Unbroken (empruntant "Luke and Leia» au Retour $d u$ Jedi) résument l'affermissement sur plusieurs films des sentiments amoureux ou fraternels qui relient certains personnages, $A$ Daring Rescue et $A$ Narrow Escape ré-exploitent quant à eux des partitions centrées sur l'action physique (respectivement "Ben's Death / Tie Fighter Attack" d'Un nouvel espoir et "The Asteroid Field» de L'Empire contre-attaque) pour mêler différentes scènes de sauvetage pour l'un et de pilotage virtuose pour l'autre. Dans l'ultime cas cité, le clip révèle le fait que la poursuite du vaisseau d'Obi-Wan par Jango Fett dans L'Attaque des clones est une reprise d'une autre séquence de la série, celle de la traversée héroïque d'un champ d'astéroïdes par le "Faucon Millénium» dans L'Empire contre-attaque. En soulignant la récurrence de certains motifs au cours de l'œuvre, plus particulièrement les similitudes entre les deux trilogies, Star Wars. A Musical Journey n'hésite pas à dévoiler les mécanismes narratifs qui sous-tendent la création de la saga, y compris ceux qui sont fondés sur le principe du recyclage 47 . Le détour par la musique permet en quelque sorte de mettre en évidence les relations fondamentales qui travaillent l'œuvre tout entière, jusque dans les schémas répétitifs qui la structurent. Plus grossièrement, cette dimension réflexive transparaît également via les animations de crayonnés, de dessins préparatoires et d'affiches qui sont insérées dans certains clips, débordant avec brutalité 


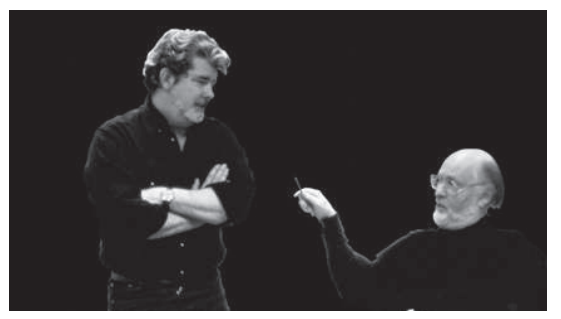

le format Cinémascope des extraits de films qu'elles côtoient pour produire des effets de surimpression, de démultiplication ou de projection vers la «caméra».48

Cette exposition décomplexée du pôle de la production renvoie clairement à la volonté de créer une confusion constante entre l'univers de la fiction et celui de l'industrie qui la génère. En témoigne le prologue de Star Wars. A Musical Journey: la fanfare de la Fox signée par Newman accompagne des photographies de Lucas et Williams prises d'abord à l'époque d'Un nouvel espoir, puis de nos jours. Le passage du noir et blanc à la couleur signale à la fois la nostalgie pour un passé disparu (en l'occurrence celui de la fin des seventies, période certes réactionnaire, mais qui rappelle l'enfance aux fans de la première heure ou représente, pour les plus jeunes, une étape importante dans l'histoire du cinéma hollywoodien) et la permanence d'une collaboration artistique exceptionnelle (l'image contemporaine des deux hommes les saisit en train de converser devant la table de mixage d'un studio d'enregistrement) (fig. 4-7). La suite de ce même segment inaugural est encore placée sous le signe de la régression vers un passé "mythique» (il s'intitule $A$ Long Time Ago). Alors que résonne le «Main Tittl», ce sont effectivement des images de la première trilogie (1977-1983) qui sont présentées à l'écran, les films les plus récents de la série n'intervenant qu'à la fin du morceau. Au moment où apparaissent les dernières harmonies du «Main Titte», la trajectoire d'Anakin est évoquée en quelques images, qui aboutissent à un gros plan inquiétant sur le visage du jeune homme ayant basculé du côté obscur. Les dissonances conclusives du morceau s'accordent alors au mouvement de sa tête encapuchonnée qui se tourne vers la caméra. Ce n'est qu'une fois rappelée cette première lecture possible de Star Wars (une expérience historique située entre 1977 et 2005, qui aboutit sur le plan diégétique à une fin tragique et désespérée) que peut s'engager l'immersion diégétique dans un univers mythique situé au-delà de l'expérience humaine du temps.

Le fait qu'A Long Time Ago, ce digest express de l'hexalogie en 1'30", ne comporte aucun dialogue n'est en rien révélateur de la démarche adoptée



7

48 Dans An Empire is Forged, cette pratique renforce indéniablement le statut d'icône médiatique de Vader; dans A Defender Emerges, l'apparition progressive des lignes représentant la figure de la princesse fait écho au mouvement même de son thème musical; enfin, dans An Unlikely Alliance, l'arrangement cheap du thème jazzy de la Cantina, tiré d'Un nouvel espoir, sert de prétexte à un ballet ouvertement grotesque où se superposent différentes esquisses de créatures monstrueuses liées à l'univers Star Wars. Signe de l'absence totale de sérieux qui caractérise ce dernier segment destiné avant tout aux fans: I'inclusion parmi la galerie monstrueuse d'un portrait de Lucas lui-même! 
49 Exception faite de quelques sons émis par les droïdes (leur "voix" en quelque sorte).

50 En 1952, Pierre Schaeffer désigne par ce terme l'audition d'un son dont la source ne peut être identifiée visuellement. Voir notamment Michel Chion, Un art sonore, le cinéma, Editions des Cahiers du cinéma, Paris, 2003, p. 411.

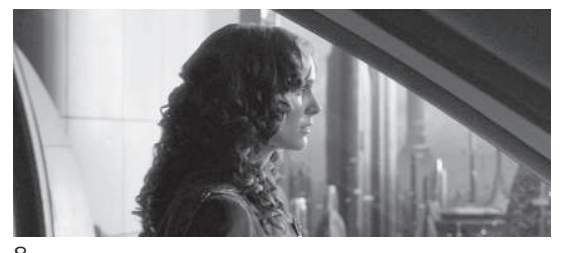

dans l'ensemble de Star Wars: A Musical Journey. Si l'éradication presque totale 49 des bruits d'ambiance apparaît comme un aveu d'impuissance face à l'antagonisme qui caractérise la relation entre sound design et piste musicale au sein de Star Wars (voir supra), la présence de paroles occupe en effet une certaine place dans les autres segments de ce DVD. Le rapport entre musique et dialogue a été pensé dès l'époque "classique" des années 1930-1940, où de nombreux compositeurs ont été animés par le souci de trouver un équivalent du chant de l'opéra qui permet la fusion entre la force poétique des mots et la puissance expressive des instruments musicaux. La précision absolue des effets de synchronisme que rendait possible l'avènement du film parlant a été perçue comme l'occasion de développer une telle interaction entre le discours verbal et les sonorités orchestrales, en faisant du film moins l'héritier du Wort-Ton-Drama wagnérien que celui du mélodrame (au sens que recouvrait ce terme au XVIII ${ }^{e}$ siècle : un drame alternant musique et déclamations, celles-ci pouvant quelquefois être accompagnées par l'orchestre). C'est pourtant essentiellement dans un rapport hiérarchique que la musique est utilisée au sein du cinéma hollywoodien (et plus largement dans les pratiques dominantes des films de fiction). Elle passe effectivement en background dès que surgit le dialogue, véhicule privilégié des informations narratives. Bien que centré sur la musique, Star Wars. A Musical Journey n'échappe pas à cette règle. Si les moments dialogués sont bien réduits à quelques brèves interventions, ils sont insérés dans les passages piano des développements musicaux. Les introductions en ostinato ou les relances précédées de silences, des procédés qu'on retrouve par exemple dans des morceaux comme "Duel of the Fates" (pour le segment Dark Forces conspire) ou "Battle of the Heroes» (pour A Hero Falls) conviennent idéalement au placement de phrases-clés puisées dans les films, avant que la musique chorale ne reprenne toute son ampleur. Les voix sont souvent détachées de leur contexte filmique, leur ton solennel résonnant d'abord sur d'autres images à valeur plus générale (villes, vaisseaux, etc.), avant de s'ancrer finalement dans des plans visualisant la source de la parole. Les différents effets liés à la synchronisation audiovisuelle sont donc utilisés, de la tension acousmatique 50 à la puissance dégagée par
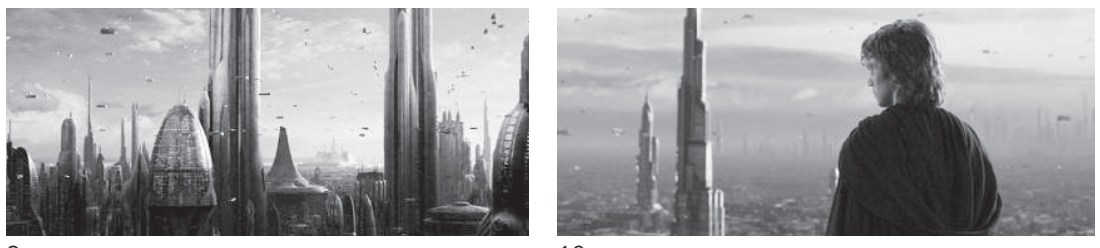

10 
l'alliance entre une expression faciale et le grain d'une voix spécifique. Quelquefois ralenties, les images extraites des films se plient continuellement aux inflexions musicales, montées en fonction des changements de tempo et de volume, ainsi qu'aux effets de modulation, de variation harmonique ou encore d'exposition thématique. Dans A Fateful Love, la partie plus agitée de la mélodie "Across the Stars» est ainsi illustrée par des plans renvoyant à la découverte de la face «sombre " d'Anakin, avant que la flamboyance retrouvée du thème ne soit associée à des séquences d'action où le couple est en péril (l'usine et l'arène de L'Attaque des clones) $\mathbf{5 1}$.

Même si nombre de ces segments audiovisuels souffrent fréquemment de leur inféodation à une musique ayant été préalablement élaborée en fonction du dynamisme spécifique à la construction spatio-temporelle précise de séquences filmiques $\mathbf{5 2}$, la plupart de ces courts métrages parviennent en fin de compte à exposer certains aspects importants de la saga en croisant les qualités propres du discours musical à un véritable effort d'épure et de synthèse au plan du drame visuel.

\section{Vers l'expansion de l'espace scénique}

Dans les six films de la série, seuls quelques moments atteignent en fin de compte cette forme de lyrisme visuel et sonore qui rappelle l'idéal musicaliste, imprégné de wagnérisme, qui a dominé les réflexions esthétiques sur le cinéma au cours de l'époque muette. Parmi ces passages les plus «rythmiques» figure assurément la conclusion du Retour du Jedi, longue séquence en montage alterné décrivant les fronts multiples - et interdépendants à divers titres - sur lesquels se déroule simultanément la bataille finale de la saga. Cette figure du montage alterné revient dans La Revanche des Sith pour exprimer des rapports intimes entre des personnages distants l'un de l'autre (le regard que paraissent échanger Padmé et Anakin, pourtant situés dans des immeubles différents) (fig. 812); pour rendre explicite l'articulation entre le pouvoir politique et les manifestations directes ou occultes de celui-ci (Palpatine au Sénat / Anakin éliminant les membres de la Fédération du Commerce); ou encore pour montrer les relations symétriques entre deux actions importantes (la naissance de Luke et Leia / l'avènement de Darth Vader; le

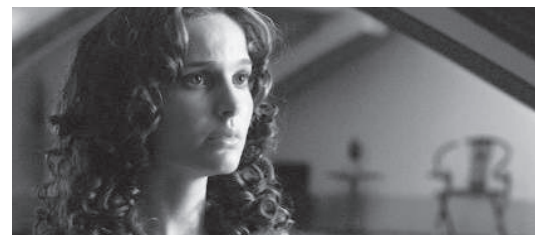

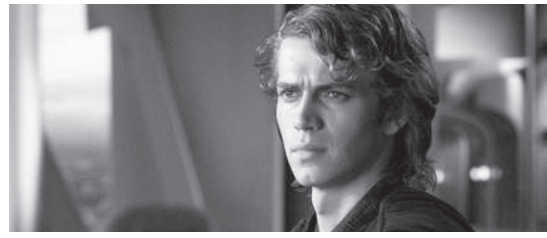

12 $\mathbf{5 1}$ Ce segment est le seul dont l'introduction de Ian McDiarmid explicite le projet au-delà de la stricte adéquation au contenu narratif, en l'occurrence le titre du morceau utilisé ("Across the Stars"): la relation amoureuse de Padmé et Anakin s'avère effectivement, selon les termes du narrateur, trop brève pour pouvoir se déployer ailleurs qu'"à travers les étoiles".

52 Prévues pour un usage extra-cinématographique, les versions de concert s'adaptent évidemment mieux à ces nouveaux montages que les passages musicaux directement tirés de la bande originale des six films.

Padmé (Natalie Portman) et Anakin (Hayden Christensen) sont progressivement reliés par l'intensité du jeu des acteurs, le travelling avant et un montage alterné qui efface l'espace urbain séparant le couple. 


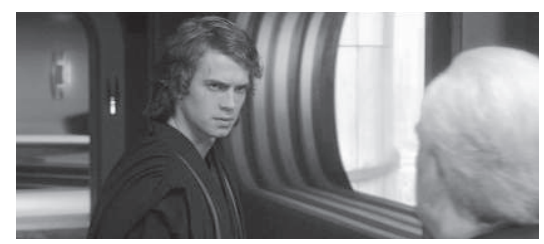

13

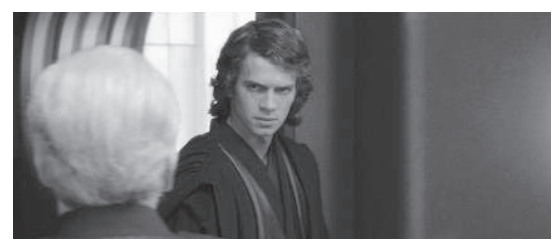

14

combat engagé d'un côté par Darth Sidious contre Yoda, et de l'autre par Anakin contre Obi-Wan). Ces séquences recourent évidemment à la musique pour amplifier le discours visuel: dans le dernier exemple cité, les deux thèmes "Duel of the Fates» et "Battle of the Heroes» sont respectivement associés à chacun des pôles de l'alternance représentée à l'écran. Déjà accentuée par leur entremêlement visuel, l'intensité dramatique concentrée dans le double duel est dès lors exacerbée par un déferlement à la fois martial et tragique des cuivres, des percussions et des chœurs.

C'est justement dans l'exploitation des possibilités du montage que se situe l'une des spécificités du spectacle cinématographique dans son rapport à l'opéra. Au moins depuis l'entre-deux-guerres, le cinéma a paru offrir des ressources inédites en termes de représentation visuelle, situées au-delà des limites spatio-temporelles de la scène. Dans cette optique, le film signalerait dès lors l'accomplissement des aspirations wagnériennes centrées sur la résurgence de la tragédie antique au cœur de la modernité technologique, ainsi que sur l'émergence d'un nouveau Gesamtkunstwerk

$\mathbf{5 3}$ Voir le chapitre 5 ("Le film, expression renouvelée du rythme collectif") de mon ouvrage L'Age du Rythme. Cinéma, musicalité et culture du corps dans les théories françaises des années 1910-1930, Payot, Lausanne, 2006. consacrant le retour aux valeurs sociales et collectives de l'art ${ }^{53}$. Dans Star Wars, cette problématique du montage comme extension de l'espace scénique s'inscrit dans une esthétique difficile à appréhender, dans la mesure où elle oscille entre la reconduction de conventions dominantes et l'introduction de figures plus expérimentales, comme le démontre la construction spatiale dans La Revanche des Sith.

Dans les passages de ce film où interagissent les figures humaines (dialogues, combats...), seuls les différents moments de coprésence entre Anakin et Palpatine bénéficient d'un mode de représentation adapté à l'évolution de leur relation: ainsi cette séquence située au milieu du film où le jeune homme accède à la révélation du pouvoir Sith

Exprimant physiquement le basculement potentiel des points de vue, les interprètes d'Anakin (Hayden Christensen) et de Palpatine (Ian McDiarmid) ne cessent d'échanger leurs positions dans l'espace.

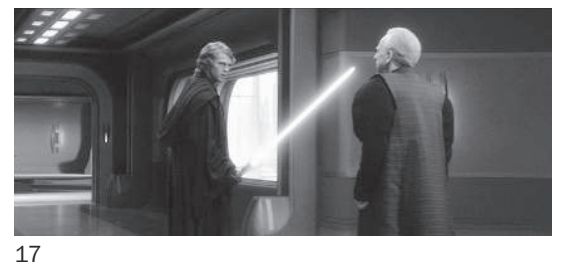




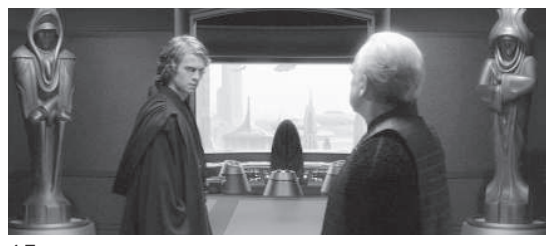

15

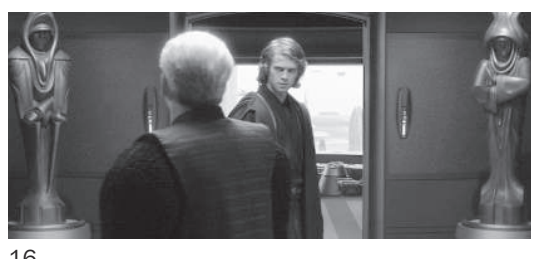

16

par Palpatine / Darth Sidious et où les deux acteurs tournoient dans un espace à $180^{\circ}$ pour exprimer le basculement possible des valeurs et des points de vue (fig. 13-14; 15-16; 17-20). A quelques exceptions près, les confrontations entre humains (dialogues et combats au sabre) de La Revanche des Sith se caractérisent par un mode de découpage très conventionnel (champ/contrechamp ou raccord dans l'axe), reflétant une défiance manifeste à l'encontre des potentialités innovatrices du montage en tant que moyen d'articuler des éléments figurant au sein du même espace - à l'opposé donc du niveau abordé ci-dessus (montage alterné entre éléments disjoints spatialement). Contrairement à une idée reçue, le rôle prépondérant accordé au numérique et à l'intertexte du jeu vidéo ne provoque dans les derniers films Star Wars aucune accélération du débit des cadres, mais aboutit au contraire à un traitement minutieux des qualités intrinsèques de l'image, par le biais de la composition visuelle ou des mouvements du cadre (peut-on encore parler de "mouvements de caméra"?). Si une partie substantielle du métrage est découpée en champs/contrechamps tout à fait standards qui mettent considérablement à mal l'ampleur souhaitée des performances et des échanges verbaux, de nombreuses images inscrivant les personnages dans les décors de synthèse (innombrables machines de guerre en déplacement dans les plans de batailles; arrière-plans vitrés sans cesse traversés par des vaisseaux...) dévoilent quant à elles un travail constant sur la superposition, voire la saturation de strates mobiles d'informations visuelles.

Cette exaspération de la relation entre le corps de l'interprète humain et la nouvelle scène numérique semble certes condamnable en vertu d'arguments similaires à ceux employés par Adorno à l'encontre de l'emphase et de l'excès fantasmagorique du spectacle wagnérien, mais

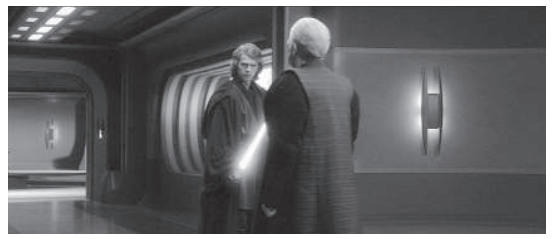

18

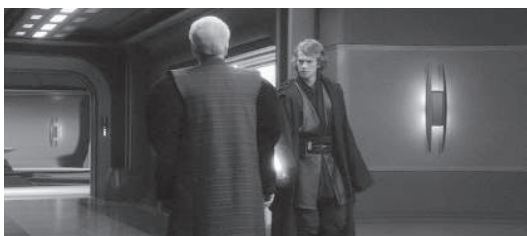

19

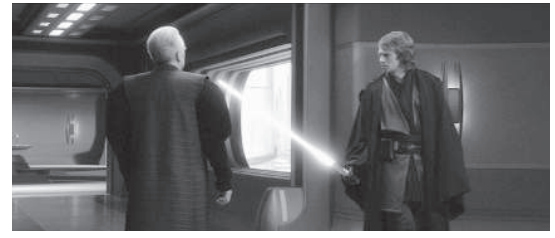

20 
L'avènement graduel d'un monde de synthèse: sous leur forme Sith, Palpatine et Anakin sont passés de la contemplation d'un spectacle à celle d'un univers artificiel

(ici l'Etoile Noire).

$54 \mathrm{~A}$ propos du "gestus social" au cinéma comme "théâtralisation directe des corps ", voir Gilles Deleuze, L'Image-Temps, Minuit, Paris, 1985, p. 250.

$\mathbf{5 5}$ Voir par exemple "The Actor and the ÜberMarionette", The Mask, vol. I, n 2, avril 1908, in Arnold Rood (éd.), Gordon Craig on Movement and Dance, Dance Horizons, New York, 1977, p. 50.



21

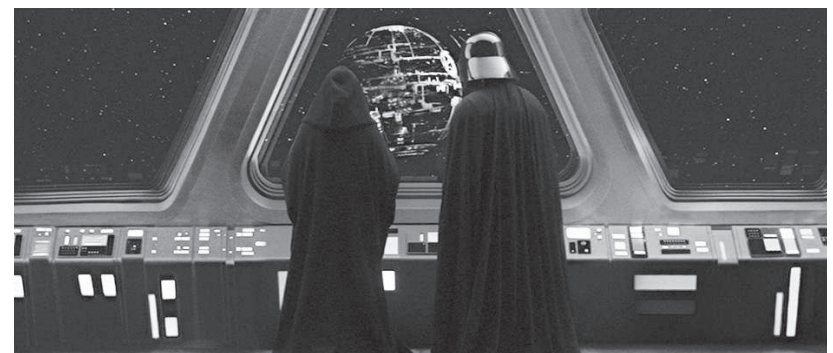

22

elle peut également apparaître comme une stylisation singulière qui mériterait d'être évaluée en regard de certains aspects du "gestus" propre à l'esthétique brechtienne de la scène ${ }^{54}$, ou encore de l'objectivation mathématique du corps réclamée par les théoriciens mécanistes (par exemple la «sur-marionnette» de Gordon Craig, en rapport rythmique constant avec son contexte plastique) 5 5. La problématique des limites de la représentation scénique est sommairement évoquée dans une séquence de La Revanche des Sith, où Palpatine invite Anakin à le rejoindre au sein d'un vaste amphithéâtre pour contempler une sorte de ballet où de gigantesques formes biologiques élémentaires évoluent dans l'espace sur le fond sonore d'un flux arythmique dans les graves. Au cours du dialogue qui s'engage à cet instant, le futur empereur commence à initier le jeune homme aux secrets du côté obscur de la Force, en insistant

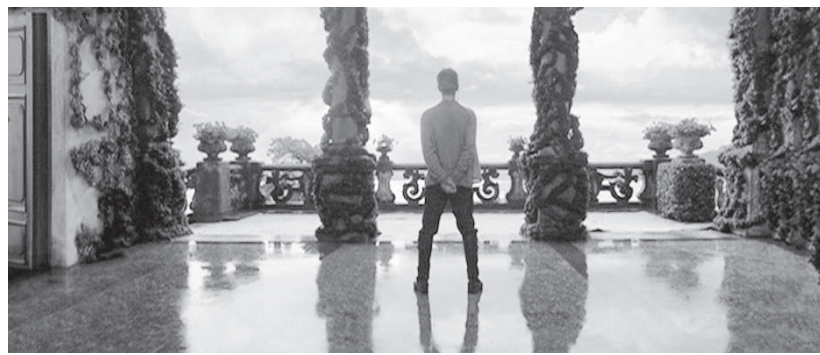

23

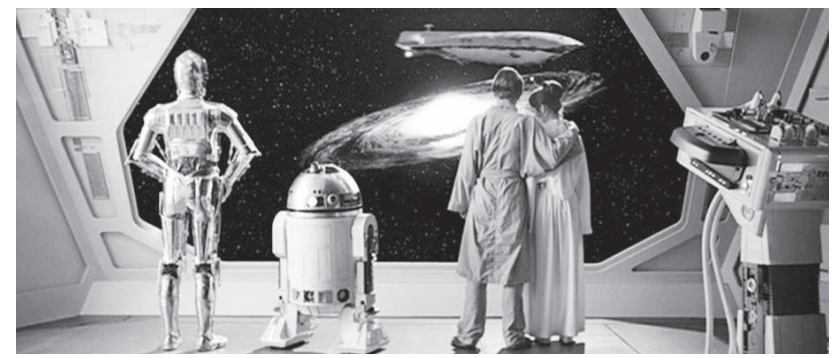

24 
notamment sur la possibilité de produire une vie artificielle. La convergence est frappante entre ce discours et le spectacle auquel assistent les protagonistes: une réorganisation mobile et abstraite du vivant à des fins purement ornementales (fig. 21-22). Lorsqu'un plan cadre les deux personnages de dos face à cette envoûtante monstruosité, s'opère le dépassement troublant d'un motif iconographique central de la culture américaine auquel ne cesse de se référer Lucas dans Star Wars: l'image idyllique de la symbiose entre l'humain et la nature via le regard porté par l'homme sur le paysage (fig. 23-26). A ce cadre fusionnel se substitue, dans cette séquence de La Revanche des Sith, la simulation esthétique d'un univers fictionnel. La représentation absolue d'un monde imaginaire, c'est en définitive ce fantasme même, autorisé par l'avènement des techniques digitales, que célèbre constamment Star Wars tout en prétendant le condamner par la reconduction de schémas narratifs et de conventions spectaculaires tout à fait traditionnels.
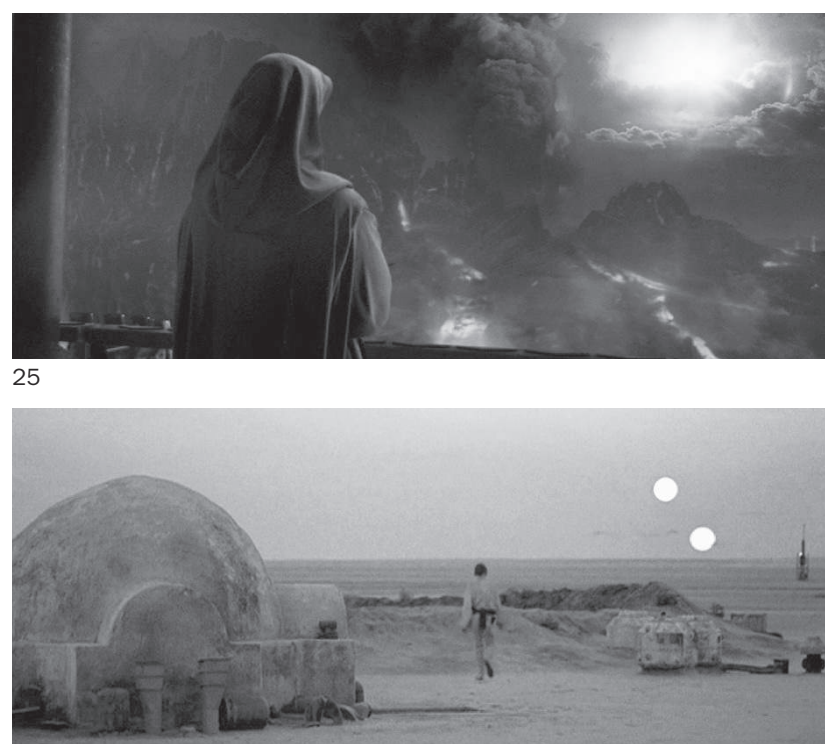